\title{
Communications
}

\section{DND-189 as an Amyloid Aggregation Probe}

\author{
Haneul Kwon, Ah Young Jee, and Minyung Lee \\ Division of Chemistry and Nanoscience, Em ha Womans Lniversity, Seoul 120-750, Korea. E-mail: myleeatewhac.kr \\ Received March 24, 2009, Accepted.April 20, 2009
}

Key Wonds: $\beta$-Amy loid peptide. Aggregation. Oligomer. Fluorescence probe

According to amy loid cascade hypothesis. $\beta$-anyloid (AB) aggregation in brain is a main cause of the pathogenesis of Alzheimer s disease. ${ }^{1} \mathrm{~A} \beta$ oligomers are highly toxic to neuron. and thus their formation and characterization have been subjected to intensive studies for many years. ${ }^{2-5}$ However. any fluorescence probe. which is specific to $A \beta$ oligomers. has not been reported yet. In this work. we first present the effective use of DND-189 as a fluorescence probe for amyloid oligomers. DND-189. which is commercially available. has been widely used as a $\mathrm{pH}$ sensor. Figure 1 shows the molecular structure of DND-189. The change of fluorescence quantum yield of DND-189 with various $\mathrm{pH}$ has been measured by the frequency modulation technique. ${ }^{6}$ They explained the lifetime change in terms of the degree of protonation to the weak base side chain. We are not aware of any time-resolved fluorescence studies on DND-189 in nonaqueous solvents or in other environments.

DND-189 was purchased from Invitrogen and $\beta$-amyloid ${ }_{1-4}$ from Biopeptide. Inc. Lyophilized $A \beta_{1.42}$ was dissolved in $100 \%$ 1,1.1,3.3,3-hexafluoro-2-propanol (HFIP). evaporated. and resuspended in DMSO. The $5 \mathrm{mM}$ stock solution was diluted at a concentration of $110 \mu \mathrm{M}$ in HEPES buffer ( $\mathrm{pH}$ 7.4). DND-189 was added into the solution at the equivalent ratio of $1: 5\left(\mathrm{DND}-189: \mathrm{A} \beta_{1}+4\right)$. The mixed solution was incubated in a water bath for 24 hr with stirring at ambient temperature. Figure 2 shows the AFM image of A $\beta$ oligomers dried on mica. showing mostly oligomers with some fibrillar stnictures. Further processes such as ultracentrifugation and fractionization lead to a narrower oligomer distribution without any fibrillar structures. ${ }^{7}$ However, such an attempt was not made here because it would not occur in wro. The fluorescence lifetime was measured by the time-correlated single photon

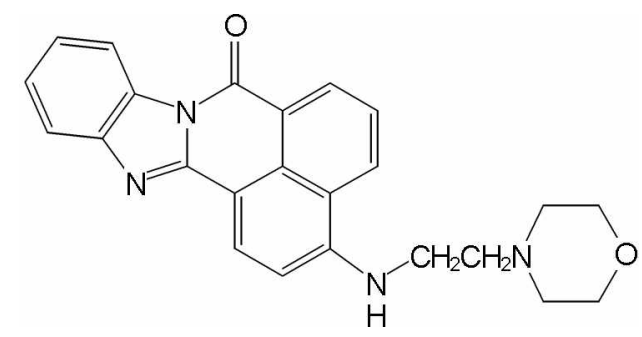

Figure 1. The molecular structure of DND-189. counting (TCSPC) systent. The sample was excited by a 467 $\mathrm{nm}$ laser beam from a picosecond diode laser operating at 20 $\mathrm{MHz}$ and the emission was collected with a fast PMT (Hamamatsu 3809-(07) and processed with a TCSPC board from Becker-Hickl (SPC-830). The fluorescence lifetimes were extracted from the measured decay curves by a nonlinear least square fit and deconvolution from the instrument response

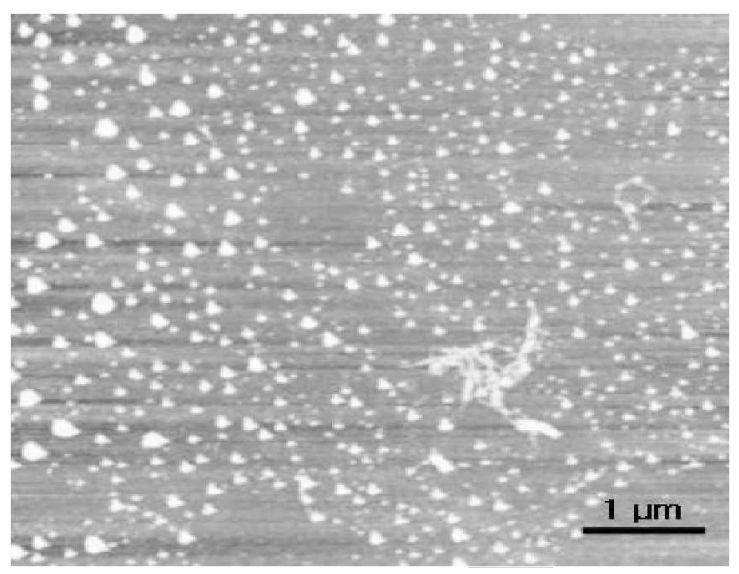

Figure 2. The AFM image of amyloid oligomers.

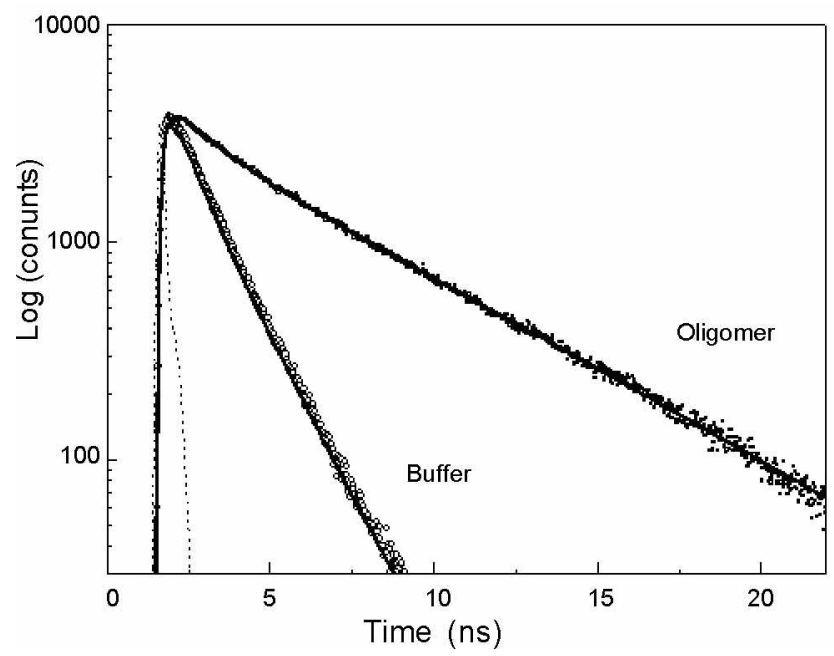

Figure 3. The fluorescence decay curves of DND-189 in buffer and in oligomers. The instrument response function was also shown. 
Table 1. The fluorescence lifetine of DND-189 in some solvents at room temperature. The dielectric constants of the solvents were also show:11.

\begin{tabular}{ccc}
\hline Solvent & Dielectric constant & Average lifetime (n1s) \\
\hline Methanol & 32.7 & 0.54 \\
Ethanol & 24.3 & 1.22 \\
Propanol & 20.1 & 3.78 \\
DMSO & 48.0 & 0.78 \\
Acetone & 20.7 & 2.75 \\
THF & 7.52 & 5.37 \\
\hline
\end{tabular}

function. For the fluorescence lifetime measurements. $A \beta$ oligomers incubated with DND-189 was diluted in HEPES buffer at the concentration of $1 \mu \mathrm{M}$. Figure 3 shows the fluorescence lifetime of DND-189 in buffer and in oligomers. The fluorescence decay curve is single exponential in buffer and becomes multiexponential in oligomers. The lifetime of DND189 was observed as 1.16 ns in HEPES buffer and $4.64 \mathrm{~ns}$ (average lifetime) in anyloid oligomers. The average lifetime was calculated by $\langle\tau\rangle=\Sigma \alpha_{i} \tau_{\text {. }}$. A slight multiexponentiality in the decay curve in oligomers seems to arise from the inhomogeniety of oligomer distribution as shown by the AFM image. We also measured the fluorescence lifetime of DND-189 in fibrils. which is almost identical to oligomers (data not shown).

The fluorescence lifetime of DND-189 was measured in some alcohols and nonaqueous solvents such as DMSO. acetone. and THF. The fluorescence decays were all multiple exponential. and their average lifetimes were shown in Table 1. The alcohol solvents were separately classified because it is known that their hydrogen bonding capability affects the excited state dynamics. It is clear that the fluorescence lifetime increases as the solvent polarity decreases. This indicates that the lifetime increase of DND-189 in A $\beta$ oligomers may be due to the polarity decrease, compared with buffer which has the higher polarity. It is also possible that the lifetime increase may be due to the rigidity in $A \beta$ oligomer environment

Thioflavin T (Th T) is most widely used for the amyloid aggregation assay. "Th T possesses high binding affinity to $A \beta$ assembly with acconpanying the shift in absorption and emission spectra to the longer wavelength. Its excitation is usually carried at $430 \mathrm{~nm}$ and enussion is recorded in the 450-600 nm wavelength. However. Th T can be only used to A $\beta$ fibrils: It does not work for oligomers. Another advantage of DND-189 over Th T is that it is a lifetime probe rather than an intensity probe. Therefore. it can be used efficiently for fluorescence lifetime imaging microscopy (FLIM) ${ }^{9} \mathrm{DND}-189$ can be excited with a longer wavelength. which holds a merit for some applications.

In conclusion, we have found a new application of lysosensor DND-189 as an amyloid aggregation probe. The average fluorescence lifetime of DND-189 increases from $1.16 \mathrm{~ns}$ in $\mathrm{pH} 7.4$ buffer to $4.64 \mathrm{~ns}$ in $\mathrm{A} \beta$ oligomers. Therefore. DND189 can be effectively used for FLIM and an amyloid oligomer assay to find new aggregation inhibitors for Alzheimer s disease therapeutics

Acknowledgments. This work was supported by the Acceleration Research Program of the Korea Science and Engineering Foundation.

\section{References}

1. Halss C.: Selkoe, D. Mol. Cell Biol. 2007, 8, 101

2. Barghom, S. et al. J. Netrochem. 2005,95, 834 .

3. Levine III, H. Amvloid 2007, $14,185$.

4. Walsh, D. M.; Selkoe, D. I. J. Newochem. 2007, 101, 1172.

5. Glabe, C. G. J. Biol. Chem. 2008, 283, 29639

6. Lin, H. J.; Heman, P.; Kang, J. S.; Lahowicz, J. R. Anal Biochem. 2001, 294, 118

7. Kim, T. E; Lee, M. Biophys. Biochent Res. Comm 2003, 303 , 576 .

8. Cser, A.: Nagy, K.; Biczok, L. Chem. Phys. Lett. 2002, $360,473$.

9. Koh, C. J.; Lee, M. Bull. Korean Chem. Soc. 2006, $27,1$. 